\author{
José Pla-Barber ${ }^{*}$ \\ Cristina Villar* \\ Ana Botella*
}

\title{
LA EXPANSIÓN INTERREGIONAL EN IBEROAMÉRICA: LA CREACIÓN DE VALOR A TRAVÉS DE FILIALES TRAMPOLÍN
}

Recientemente, desde el sector empresarial han surgido diferentes afirmaciones destacando el valor intangible que España podría ofrecer como «trampolín» en el proceso de expansión interregional de las multinacionales europeas hacia Latinoamérica. En este estudio dotamos de contenido teórico y empírico a esta realidad, ofreciendo una visión general de las características de las filiales trampolín mediante una muestra de 188 filiales localizadas en España. Con este trabajo pretendemos aportar directrices a la práctica empresarial que faciliten la legitimidad en sus inversiones en otras regiones, especialmente en países emergentes donde existe un mayor riesgo de pérdida de valor.

\section{Interregional expansion in Iberoamerica: value creation through springboard subsidiaries}

Recently, from the business sector different claims have emerged highlighting the intangible value that Spain could offer as a "springboard" in the process of interregional expansion of European multinationals towards Latin America. In this study we provide theoretical and empirical content to this reality, offering a general overview of the profile of the springboard subsidiary and its characteristics through a sample of 188 subsidiaries located in Spain. We provide guidance to business practice facilitating legitimacy in their investments in foreign regions, particularly in emerging countries where there is a greater risk of loss of value.

Palabras clave: filial trampolín, multinacionales, Latinoamérica, expansión interregional, conocimiento experiencial.

Keywords: springboard subsidiary, multinationals, Latin America, interregional expansion, experiential knowledge.

JEL: F23, L22, L25, M10, M16.

\footnotetext{
* Departamento de Dirección de Empresas «Juan José Renau Piqueras». Facultad de Economía. Universidad de Valencia.

Los autores agradecen la financiación recibida por el Ministerio de

Educación y Ciencia a través del proyecto ECO2017-85456-R.

Versión de junio de 2019.

DOI: https://doi.org/10.32796/ice.2019.909.6894
} 


\section{Introducción}

Durante las últimas décadas el auge espectacular de la presencia de multinacionales españolas en Latinoamérica ha generado unas fuertes conexiones a nivel empresarial entre ambos continentes y estas relaciones, a su vez, están convirtiéndose en un factor esencial para la atracción de inversión directa hacia España en ambos sentidos: las empresas multilatinas que pretenden dar el salto a Europa y, lo que es más significativo, las empresas multinacionales globales que buscan desarrollarse en los crecientes mercados latinoamericanos.

Algunos datos macroeconómicos dan soporte a estas afirmaciones. Por un lado, la inversión directa europea en los mercados latinoamericanos está dominada en gran medida por España, que representa el $29 \%$ de la inversión europea en nuevos proyectos en la región y el $29 \%$ del total de fusiones y adquisiciones europeas por valor en el período 2005-2017 (ECLAC, 2018). Por otro lado, España es uno de los principales destinos para la inversión directa latinoamericana, siendo el segundo país receptor de esta inversión fuera de la región después de Estados Unidos. La inversión directa latinoamericana en España alcanzó los 39.490 millones de euros en 2017, y está compuesta por una multitud de empresas que operan en una gran variedad de sectores económicos (Global LATAM, 2018).

En la práctica empresarial son muchas las multinacionales europeas que reconocen expresamente las ventajas de utilizar España como país trampolín para invertir en América Latina. Empresas tan conocidas como, por ejemplo, las alemanas Thyssen, Bertelsmann o Basf, las francesas AXA, Saint-Gobain o Capgemini, la sueca Securitas o las danesas Vestas y Bestseller utilizan sus filiales en España para iniciar, desarrollar y gestionar operaciones en América Latina (ICEX, 2019). Más recientemente, se observa que este fenómeno incluso ocurre en el sentido opuesto: algunas multilatinas seleccionan España como su primer destino europeo a partir del cual orientan su expansión europea. Empresas como las colombianas Totto, Leonisa o Carvajal, la argentina Arcor, las mexicanas Bimbo, Gruma o Cemex, o las peruanas Ajegroup o Yanbal entraron en el mercado español con ese objetivo.

No obstante, a pesar del enorme interés de este fenómeno económico, el debate académico al respecto y el análisis de este en un marco teórico apropiado es más bien escaso. En este sentido nuestro trabajo pretende, por una parte: i) dotar de contenido teórico a esta realidad, y por otra ii) caracterizar a las filiales que asumen este rol de trampolín en el proceso de expansión interregional hacia Latinoamérica y poner de manifiesto las implicaciones y oportunidades que surgen para estas. ¿Cuáles son las condiciones que un país debe tener para asumir este papel de trampolín? ¿Cómo ayudan las filiales trampolín a superar las desventajas de ser extranjero en la región? ¿Cómo generan valor estas filiales en el entramado de la multinacional? Estas son algunas de las preguntas que tratamos de responder en este artículo.

A nivel teórico, nuestro estudio realiza una importante contribución a la teoría sobre el proceso de internacionalización (Johanson y Vahlne, 2009) al evidenciar cómo la internacionalización puede facilitarse no solo a través de la experiencia en el país de destino sino también a partir de cierto conocimiento experiencial que otras unidades de la organización pueden tener sobre las regiones de destino (en este caso Latinoamérica). Del enfoque exclusivo de la teoría centrado en la experiencia en un país pasamos a una visión más amplia, en la que damos un mayor protagonismo a las redes de relaciones y al conocimiento experiencial sobre las regiones. Adicionalmente, desde la perspectiva de recursos y capacidades (Grant, 1996) mostramos cuáles son los recursos y competencias específicos y los mecanismos que permiten convertir una ventaja de localización en una auténtica ventaja específica de la filial (Rugman y Verbeke, 2003). A nivel práctico, el estudio ofrece numerosas recomendaciones para la práctica empresarial al dar respuesta a una importante cuestión en el área de 
la gestión: ¿cómo puede mejorarse la implantación interregional? La filial trampolín — situada en una posición intermedia entre el país de destino y el país inversor en términos de distancia institucional y cultural — liderará inicialmente el proceso, limitará en cierta medida el riesgo y podrá favorecer el establecimiento de «legitimidad externa» en los mercados locales.

Para cumplir estos objetivos, hemos estructurado el artículo en dos partes claramente diferenciadas. En una primera parte desarrollamos un marco teórico propio y original, en el que a partir de las aportaciones de la teoría del proceso de internacionalización y de la teoría de recursos y capacidades definimos nuestro concepto de filial trampolín y su aplicabilidad en los procesos de expansión interregional. En la segunda parte desarrollamos un estudio empírico sobre una base de datos original, de la que hemos extraído tanto información primaria como secundaria. El estudio ofrece una descripción amplia del fenómeno y caracteriza los rasgos distintivos de un grupo de filiales trampolín que gestionan operaciones en Latinoamérica. El trabajo finaliza ofreciendo las principales conclusiones del estudio y toda una serie de recomendaciones relevantes para la práctica empresarial.

\section{Marco conceptual}

\section{La filial trampolín en el contexto de la teoría del proceso de internacionalización}

En el estudio de la empresa multinacional existe un renovado interés por el análisis de las filiales como auténticas protagonistas de la internacionalización (Birkinshaw, Bouquet y Ambos, 2007; Chakravarty et al., 2017). Las filiales, lejos de ser herramientas al servicio de la casa matriz con un rol determinado y definido por esta, pueden configurarse como unidades semiautónomas con sus propios recursos y poder utilizables para estimular cambios, innovaciones y crecimiento dentro de la red corporativa. Al mismo tiempo, a medida que la multinacional crece en tamaño y aumenta su complejidad, los roles y las funciones de la casa matriz tienden a diluirse en diferentes centros de la organización, tradicionalmente, en las casas matrices regionales; incluso recientemente se observa cómo estas funciones y responsabilidades pueden ser asumidas también por filiales locales que generan un alto valor añadido (Alfoldi, Clegg y McGaughey, 2012), como es el caso de las «filiales trampolín».

La definición de «filial trampolín» se enmarca en el ámbito de la teoría del proceso de internacionalización (Johanson y Vahlne, 1977; 2009). El argumento básico de esta teoría es que el conocimiento y el aprendizaje en los mercados externos son recursos imprescindibles para el desarrollo internacional de la empresa y que dicho conocimiento se adquiere a través de la acumulación de experiencia de la propia empresa en el país de destino. La internacionalización a través de una «filial trampolín» se presenta como una nueva forma de adquirir parte de este conocimiento experiencial necesario para abordar un nuevo mercado sin la necesidad inicial de invertir en este. En este sentido, dicha filial debe localizarse en un país «trampolín» situado en una posición intermedia en términos de distancia cultural e institucional entre el país de destino y el país de origen y desde el que exista un intenso flujo comercial con el país de destino. De esta forma, el aprendizaje de la casa matriz en la filial trampolín pueda trasladarse, de alguna manera, al país de destino y, por tanto, favorecer el establecimiento de «legitimidad» en dicho mercado; asimismo, dado el intenso flujo comercial con el país de destino, la empresa multinacional pueda adquirir "conocimiento empresarial» y desarrollar redes en el país de destino desde el país trampolín (Pla-Barber y Camps, 2012). Como se observa en esta definición, la condición de «país trampolín» determina unas ventajas de localización; ahora bien, estas ventajas de localización no garantizan el éxito. Son las propias filiales quienes deben reconocer dichas ventajas de localización e incorporarlas en sus estrategias. Es aquí donde juegan un papel primordial las capacidades que la filial pueda desarrollar para aprovechar dichas ventajas, convertirlas en activos específicos (Rugman y Verbeke, 2001) y hacerlos visibles 
para el resto de la organización (Birkinshaw et al., 2007). En nuestro contexto, las filiales de empresas multinacionales localizadas en España que son capaces de aprovechar estas ventajas de localización y reivindicar este nuevo rol de mayor valor dentro del entramado complejo de sus casas matrices son denominadas «filiales trampolín» (Pla-Barber y Camps, 2012).

\section{La expansión interregional}

Las filiales trampolín son particularmente relevantes en los procesos de expansión interregional (Pla-Barber, Villar y Madhok, 2018). La expansión interregional es una estrategia compleja ya que las empresas se enfrentan a las «desventajas de ser foráneas en la región» (liability of interregional foreignness) (Asmussen y Goerzen, 2013; Qian et al., 2013), lo que implica complicaciones adicionales al propio hecho de ser foráneo en el país (liability of foreignness) y foráneo en las redes de negocios (liability of outsidership) (Johanson y Vahlne, 2009). Estas empresas tienen que afrontar nuevos comportamientos en la demanda de los clientes, proveedores y otros agentes locales; y sobre todo, un incremento sustancial en las diferencias culturales e institucionales (Qian et al., 2013). Desde el punto de vista del paradigma explotación-exploración (March, 1991), mientras que en la expansión intrarregional las empresas pueden explotar la base de conocimientos preexistente, la expansión interregional supone un cambio esencial ya que el stock de conocimiento específico de la empresa tiene que modificarse sustancialmente para hacer frente a «la desventaja de ser extranjero en la región». En este sentido, la especificidad del contexto limita sustancialmente las capacidades de sus casas matrices para generar valor (Campbell y Szulanski, 2016). A medida que incrementa la distancia geográfica, cultural e institucional entre las casas matrices y las unidades locales, las funciones clave que desempeñan estas como son, por ejemplo, distribuir los recursos entre las diferentes unidades de negocio, diseñar estándares para coordinar y controlar las actividades y facilitar sinergias y aprendizaje tienden a ejecutarse incorrectamente. Por tanto, las empresas multinacionales deben diseñar soluciones organizativas que, de alguna forma, les ayuden a mitigar dichas barreras. La evidencia empírica más clásica muestra que para superar dichos inconvenientes en los procesos de expansión interregional las empresas multinacionales utilizan a menudo estructuras intermedias de gestión (Forsgren, Holm y Johanson, 2005). En la mayoría de los casos, estas estructuras intermedias se configuran a través de acuerdos de cooperación o adoptan la forma de oficinas o casas matrices regionales. Una alternativa potencial frente a la falta de conocimiento específico del contexto por parte de las casas matrices sería utilizar el conocimiento que pudiera existir en otras partes de la organización. Es aquí donde surgen las «filiales trampolín»: filiales fuera de la región pero que tienen cierto «conocimiento experiencial» en la región y que, al menos, al principio del proceso de expansión interregional pueden asumir responsabilidades sobre otras filiales localizadas en otros países del lugar de destino.

La filial trampolín puede considerarse un tipo especial de filial cualificada con un amplio alcance de mercado $y$ de valor: un centro de excelencia que juega un papel destacado en la multinacional al actuar como un jugador que integra (Gupta y Govindarajan, 1991) el entorno del país de acogida y el resto de la corporación. Como en el caso de la típica filial centrada en el mercado local desarrolla estrechos vínculos con empresas e instituciones del país de acogida, pero no para adaptarse a las especificidades locales, sino porque ello le permite desarrollar competencias que contribuyen a la ventaja competitiva de la multinacional a nivel global. En este sentido, actúa como una estructura intermedia que ejerce un doble rol: por una parte, es una filial de la casa matriz, pero al mismo tiempo actúa como casa matriz en sus relaciones con las filiales latinoamericanas. Mientras que las casas matrices se sitúan en la región de destino, estas filiales trampolín actúan como casas matrices desde fuera de la región. En el primer caso las diferencias institucionales y culturales entre las dos regiones persisten; en el 
segundo, la filial trampolín actúa como un puente en la intersección entre las dos regiones (Pla-Barber, Villar y Madhok, 2018). Así, la filial trampolín opera como vehículo transmisor de las rutinas y prácticas organizativas desde la casa matriz hacia las filiales sobre las que ejerce influencia, pero a su vez también aprende de estas y transmite dicho conocimiento a la matriz, lo que permite mejorar la «consistencia interna» (Forsgren, Holm y Johanson, 2005). Adicionalmente, la filial trampolín también puede generar nuevo conocimiento o incluso adaptarlo al contexto específico de los entornos locales (Harzing y Noorderhaven, 2006).

\section{Características de la filial trampolín: hipótesis}

Desde la perspectiva de la teoría de recursos y capacidades aplicada al ámbito de las filiales, Verbeke y Yuan (2013; 2018) argumentan que una dotación superior de recursos organizativos a nivel de filial antecede a la asignación de un mandato superior. En este sentido, tanto los recursos como las competencias específicas que una filial posea se han considerado como determinantes críticos de los roles que estas filiales pueden asumir y de su posible evolución en el entramado de la multinacional (Birkinshaw, 1997). En nuestro caso particular, con relación a los recursos, nos centramos en el conocimiento específico que las filiales trampolín tienen del mercado Latinoamericano y en la dotación de recursos financieros y directivos que la filial puede aplicar más allá de sus necesidades en el mercado local. Con relación a las competencias, analizamos el alcance de la cadena de valor de la filial trampolín, tanto a nivel de actividades como de países así como la capacidad para ser autónomas y la capacidad de generar conocimiento y competencias útiles para el resto de su organización.

\section{Recursos}

Tal y como hemos indicado en el subapartado anterior, en términos de recursos intangibles, poseer un conocimiento específico sobre la región objetivo (es decir, el conocimiento del mercado regional) sería una de las condiciones necesarias para desarrollar un rol de trampolín (Pla-Barber y Camps, 2012). Este conocimiento incluiría tanto el conocimiento institucional (conocimiento que una empresa posee de la cultura del país, de su lengua, tradiciones, normas de comportamiento, sistema educativo, leyes, regulaciones y costumbres comerciales) como el conocimiento empresarial (el conocimiento de los posibles clientes, competidores y condiciones del mercado en un país concreto). Este conocimiento de la filial trampolín incorpora conocimiento tácito (difícilmente codificable) y específico del contexto local. Al mismo tiempo, si este conocimiento se percibe por la casa matriz y el resto de filiales como único y claramente diferenciado, la filial puede convertirse en un centro de referencia al que asignar más recursos y dotar de una mayor autonomía. Ambos aspectos son esenciales para desarrollar el «rol de trampolín», ya que este requiere del potencial para formular estrategias e implementar decisiones autónomas en los países o regiones objetivo. Por tanto, planteamos la siguiente hipótesis:

H1: Las filiales trampolín muestran un grado más elevado de conocimiento experiencial en Latinoamérica respecto a otras filiales que no asumen este rol.

Con relación a los activos tangibles, la filial trampolín necesita gestionar operaciones no solo en su país de origen sino también en otras países, por tanto, requiere de recursos financieros y directivos sobrantes, "holgura organizativa», de forma que puedan asignarse flexiblemente allá donde sean necesarios. Esta disponibilidad de recursos es clave para evitar el riesgo de que las responsabilidades gerenciales en los mercados de inversión afecten a la gestión del propio mercado (Birkinshaw, 1997). Estos recursos sobrantes en muchas ocasiones se convierten en el catalizador de iniciativas por parte de las filiales (Verbeke y Yuan, 2013), como podría ser la inversión en Latinoamérica. 
La «disponibilidad de recursos directivos» es específica a la propia empresa, y por tanto sus directivos preferirán vías de expansión dentro de los límites en los que están acostumbrados, en los cuales puedan aplicarse fácilmente las rutinas existentes (Mishina et al., 2004). Capacidades directivas ociosas en un país intermedio y alineadas con las rutinas existentes pueden dedicarse a acumular nuevo conocimiento del entorno y a construir enlaces con las redes locales en la región de destino. Por lo tanto, aquellas filiales que cuentan con cierta holgura directiva y están ubicadas en un país intermedio serán más eficaces en la supervisión de las operaciones en la región objetivo.

De acuerdo a ello:

H2a: Las filiales trampolín muestran una mayor disponibilidad de recursos directivos respecto a otras filiales que no asumen este rol.

La disponibilidad de recursos financieros reduce el nivel de preocupación sobre las operaciones en el extranjero ya que las empresas no están presionadas por deudas importantes, al tiempo que disponen de ciertos recursos sobrantes para poder «experimentar» con nuevas iniciativas. Del mismo modo, la holgura financiera se ha relacionado positivamente con la mayor asunción de los riesgos que conlleva la inversión en otras regiones (Lin et al., 2009). Por tanto:

H2b: Las filiales trampolín muestran una mayor disponibilidad de recursos financieros respecto a otras filiales que no asumen este rol.

\section{Competencias}

El alcance de la filial en términos de actividades de la cadena de valor y mercado es un indicador de su nivel de competencias, de tal manera que las filiales tienen un alcance de la cadena de valor especializado si realizan una gama limitada de actividades, ya sea «hacia arriba» (producción y/o abastecimiento) o «hacia abajo» (ventas y marketing) (Andersson, Forsgren y Holm, 2007). Si bien un enfoque limitado de las actividades de la cadena de valor tiene claras ventajas para la especialización de la base de conocimiento de la filial, también restringe su capacidad de absorción, ya que el reconocimiento, la asimilación y la aplicación del conocimiento se relacionará únicamente con las actividades que la filial realiza (Schleimer y Pedersen, 2014). En este sentido, un amplio alcance en las actividades de la cadena de valor aumenta el stock de conocimiento necesario para gestionar y coordinar otras unidades que pueden tener diferente naturaleza. Se produce cierto ajuste del conocimiento entre la filial trampolín y las necesidades de las filiales, de forma que las directrices, que emanan de la filial trampolín y de la propia casa matriz, pueden trasladarse, entenderse y absorberse más fácilmente (Asmussen, Foss y Pedersen, 2013). Por tanto:

H3a: Las filiales trampolín desarrollan un mayor número de actividades distintas de la cadena de valor (menor especialización) respecto a otras filiales que no asumen este rol.

Por otra parte, a nivel de filial, su alcance geográfico es un indicador del rol desempeñado. De hecho, Birkinshaw y Hood (2000) demostraron cómo un amplio alcance internacional era un antecedente para la inversión en clústeres de alta innovación. Las filiales con un alcance geográfico que va más allá de las fronteras del país de origen están acostumbradas a tratar la heterogeneidad del entorno (por ejemplo, diferencias en la dinámica competitiva, estructuras de mercado o canales de distribución) y están expuestas a diversas oportunidades que la casa matriz podría aprovechar para asignarles un rol de mayor valor añadido (Hoenen et al., 2014). Por lo tanto, proponemos la siguiente hipótesis:

H3b: Las filiales trampolín muestran un alcance geográfico más elevado (presencia en mayor número de mercados) respecto a otras filiales que no asumen este rol. 
La capacidad de la filial para incorporar las ventajas de su localización a su estrategia depende del nivel de autonomía que ostente en la toma de decisiones. La razón es que una baja autonomía indica un alto nivel de control burocrático por parte de la casa matriz reduciendo el espacio para la iniciativa y emprendimiento (Birkinshaw, 1997). Por el contrario, un elevado nivel de autonomía es un atributo indispensable para la filial trampolín ya que la dota con más margen para la exploración y una mayor habilidad para desarrollar redes más extensas (Birkinshaw, Hood y Johnson, 1998), así como una mejor adaptación a entornos distintos por la flexibilidad que le aporta (Cantwell y Mudambi, 2005). Por tanto proponemos:

H3c: Las filiales trampolín muestran un grado de autonomía estratégica más elevado respecto a otras filiales que no asumen este rol.

Por último, múltiples estudios relacionan la capacidad de las filiales para generar valor en la empresa con el desarrollo de mandatos relevantes en la multinacional. Por ejemplo, la literatura sobre centros de excelencia establece que, más allá de la capacidad para generar nuevas competencias, los centros de excelencia son reconocidos como tal por ser capaces de adaptar ese nuevo conocimiento para que pueda ser usado por otras unidades de la empresa (Andersson y Forsgren, 2000; Frost, Birkinshaw y Ensign, 2002). De la misma manera, la filial trampolín debe ser capaz, no solo de almacenar el conocimiento sobre otros contextos, sino también de poder hacerlo útil para el resto de la organización (Gupta y Govindarajan, 2000) y ser reconocida por ello. A la transformación de nuevo conocimiento en conocimiento útil por el resto de la compañía se le denomina capacidad de creación de competencias únicas (Palmié, Keupp y Gassman, 2014). Por tanto, proponemos la última hipótesis:

H3d: Las filiales trampolín muestran un grado de creación de competencias únicas más elevado respecto a otras filiales que no asumen este rol.

\section{Estudio empírico}

\section{Población objeto de estudio, muestra y recolección de datos}

Al objeto de comprobar a nivel empírico el uso de este rol, sus principales características y antecedentes, nuestro estudio utiliza dos metodologías diferentes pero complementarias a la vez.

En primer lugar, a través de la base de datos Orbis, identificamos todas las filiales que pudieran considerarse trampolín, sobre la cuales realizamos un estudio descriptivo de sus características. El criterio de selección se estableció de la siguiente forma: filiales localizadas en España que fueran propiedad de una empresa multinacional (con un porcentaje de propiedad superior al $50 \%$ ) y que, al menos, tuvieran, a su vez, una filial en Latinoamérica (con un porcentaje de propiedad superior al $25 \%$ ). Los porcentajes de propiedad no son aleatorios. En el primer caso, consideramos que una propiedad mayor del $50 \%$ supone que la filial española está controlada y forma parte de la estrategia desarrollada por el grupo multinacional. Por otra parte, la función de trampolín puede realizarse no solo a través de la propiedad directa sino también a través de relaciones indirectas que no impliquen propiedad. Por este motivo, en este caso relajamos la necesidad de tener más de un $50 \%$ de control, aunque como se comprueba a partir de los datos, el porcentaje de control de la filial española en la mayoría de los casos tiende a ser muy superior al $25 \%$. Esta búsqueda proporcionó una muestra de 155 filiales trampolín que controlan a su vez 879 filiales en Latinoamérica.

En segundo lugar, una vez identificadas las empresas trampolín, incorporamos un grupo de control de empresas filiales localizadas en España, cuyas casas matrices también tenían inversiones en Latinoamérica, pero dichas inversiones no eran gestionadas/controladas por las filiales españolas.

Con el objeto de obtener información primaria, se envió un cuestionario a ambos grupos desde diciembre 


\section{GRÁFICO 1}

\section{ANTIGÜEDAD Y TAMAÑO DE LAS FILIALES TRAMPOLÍN}

(En \%)
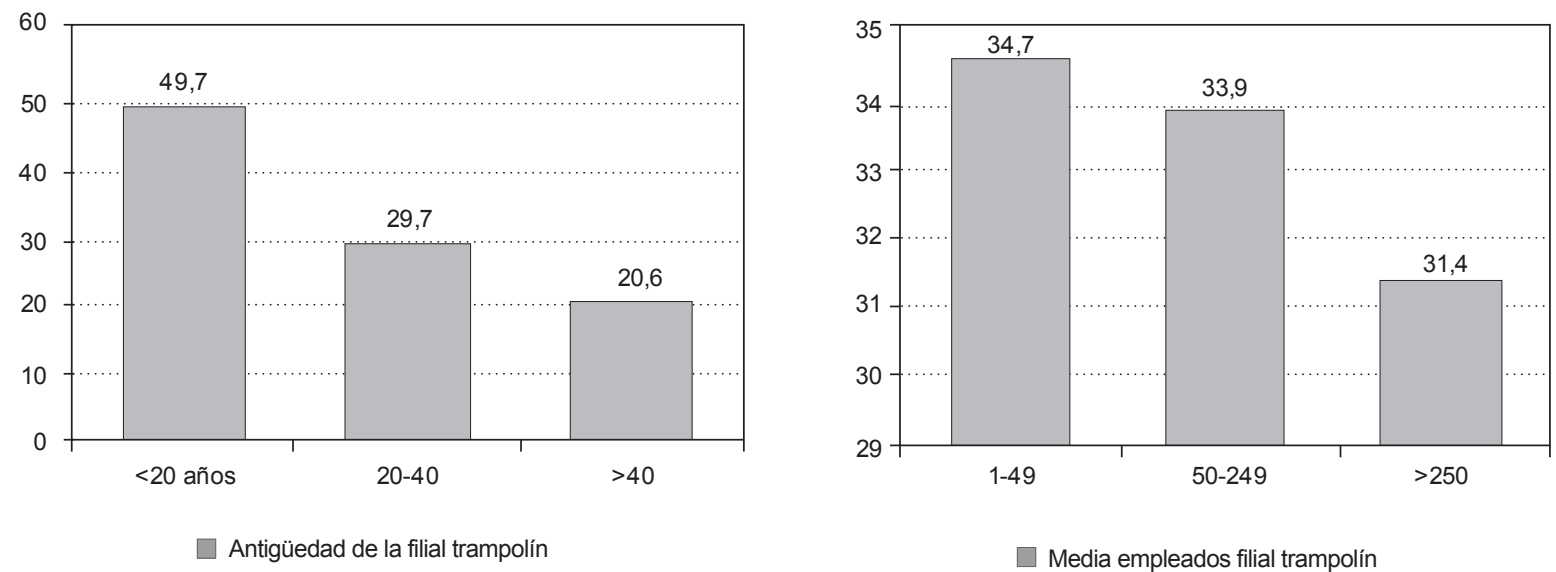

FUENTE: Elaboración propia.

de 2016 a diciembre de 2017. En esta parte del estudio, las respuestas obtenidas fueron 188 , de las cuales 69 correspondían a filiales trampolín y 119 al grupo de control. El cuestionario incluía variables medidas en escalas Likert de cinco y siete puntos. Adicionalmente esta información se complementó con datos objetivos publicados de cada una de las empresas y con la base de datos Orbis, utilizada para contrastar las hipótesis relativas a los antecedentes del rol.

\section{Análisis descriptivo}

\section{Tamaño, experiencia y control}

Como puede observarse en el Gráfico 1 las filiales trampolín suelen ser filiales consolidadas tanto a nivel de experiencia como de tamaño. Generalmente, este tipo de filiales que han desarrollado sus propias redes en el exterior son filiales expertas que han acumulado una serie de capacidades importantes para la casa matriz a lo largo de su historia, si bien el rol que desempeñan varía con el tiempo. En ocasiones, estas filiales trampolín se convierten en la casa matriz para Latinoamérica de la empresa multinacional porque se ven envueltas en procesos de adquisiciones a nivel internacional donde la multinacional busca no solo comprar la filial española sino la red que tenía previamente desarrollada en Latinoamérica. Prácticamente un $50 \%$ de las empresas de la muestra tienen hasta 20 años, casi un $30 \%$ tiene entre 20 y 40 años y un $20 \%$ más de 40 años. En consecuencia, el tamaño también tiende a ser relativamente más grande que las filiales que operan sobre una base local. Basándonos en la media de empleados de los tres últimos años, en total un $68 \%$ de las filiales trampolín tienen más de 50 empleados, y casi un $32 \%$ más de 250 empleados.

Como unidades en las que se delega la implementación de la estrategia internacional en Latinoamérica, las filiales trampolín poseen un elevado control sobre las filiales en el mercado de destino. Si bien el criterio de control se fijaba inicialmente en un mínimo del $25 \%$, como se puede observar en el Gráfico 2 en la realidad las 


\section{GRÁFICO 2}

FILIALES EN LATINOAMÉRICA PROPIEDAD DE LA FILIAL TRAMPOLÍN (En \%)

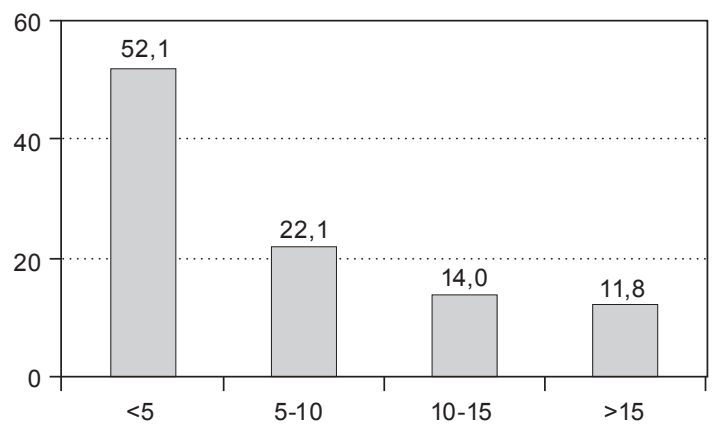

$\square$ Número de filiales en Latinoamérica

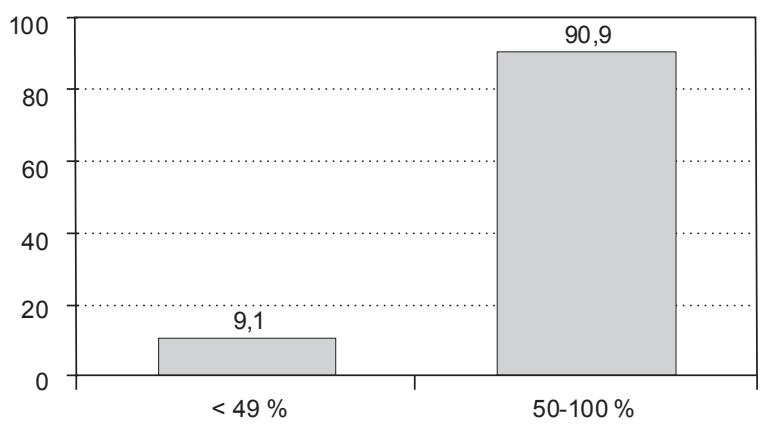

$\square \%$ de propiedad sobre las filiales en Latinoamérica

FUENTE: Elaboración propia.

filiales trampolín ejercen un control prácticamente total sobre sus filiales latinoamericanas, con un porcentaje medio de propiedad sobre estas que llega a ser mayor del $90 \%$. En el Gráfico 2 se presenta también el número total de filiales en Latinoamérica contraladas por las filiales trampolín. Más del $50 \%$ de la muestra posee en Latinoamérica hasta cinco filiales, y un $22 \%$ entre cinco y diez, mientras que en total un $25 \%$ tiene más de diez. Así, esto nos indica que cuando una filial asume este rol generalmente se sigue un proceso de expansión acumulativo.

\section{Distribución sectorial}

Del total de filiales trampolín, el $70 \%$ pertenecen a sectores de prestación de servicios y el $30 \%$ a sectores de fabricación. El Gráfico 3 ilustra la distribución de estas filiales de acuerdo al grado de intensidad tecnológica de sus respectivos sectores.

Como se puede observar, las ventajas de las filiales trampolín son mucho más visibles en el sector de servicios que en los sectores manufactureros. Los aspectos relacionados con el conocimiento institucional son más relevantes en servicios (cultura, idioma, valores, etc.) porque básicamente dependen de la interacción personal. Además, en el caso particular de la relación de España con Latinoamérica podemos encontrar una serie de sectores especialmente atractivos en los que se puede aprovechar claramente el conocimiento empresarial: $i$ ) sectores relacionados con la cultura, la educación y la comunicación y ii) sectores en los que las empresas españolas se han convertido en líderes mundiales, que incluyen industrias de servicios tradicionales como la banca, telecomunicaciones y energía, y otras cuyo auge es más reciente como es el caso de las energías renovables y la gestión de los servicios públicos e infraestructuras.

\section{Análisis cuantitativo}

En esta parte del análisis pretendemos contrastar las hipótesis planteadas con relación a los recursos y competencias que caracterizan las filiales trampolín. 


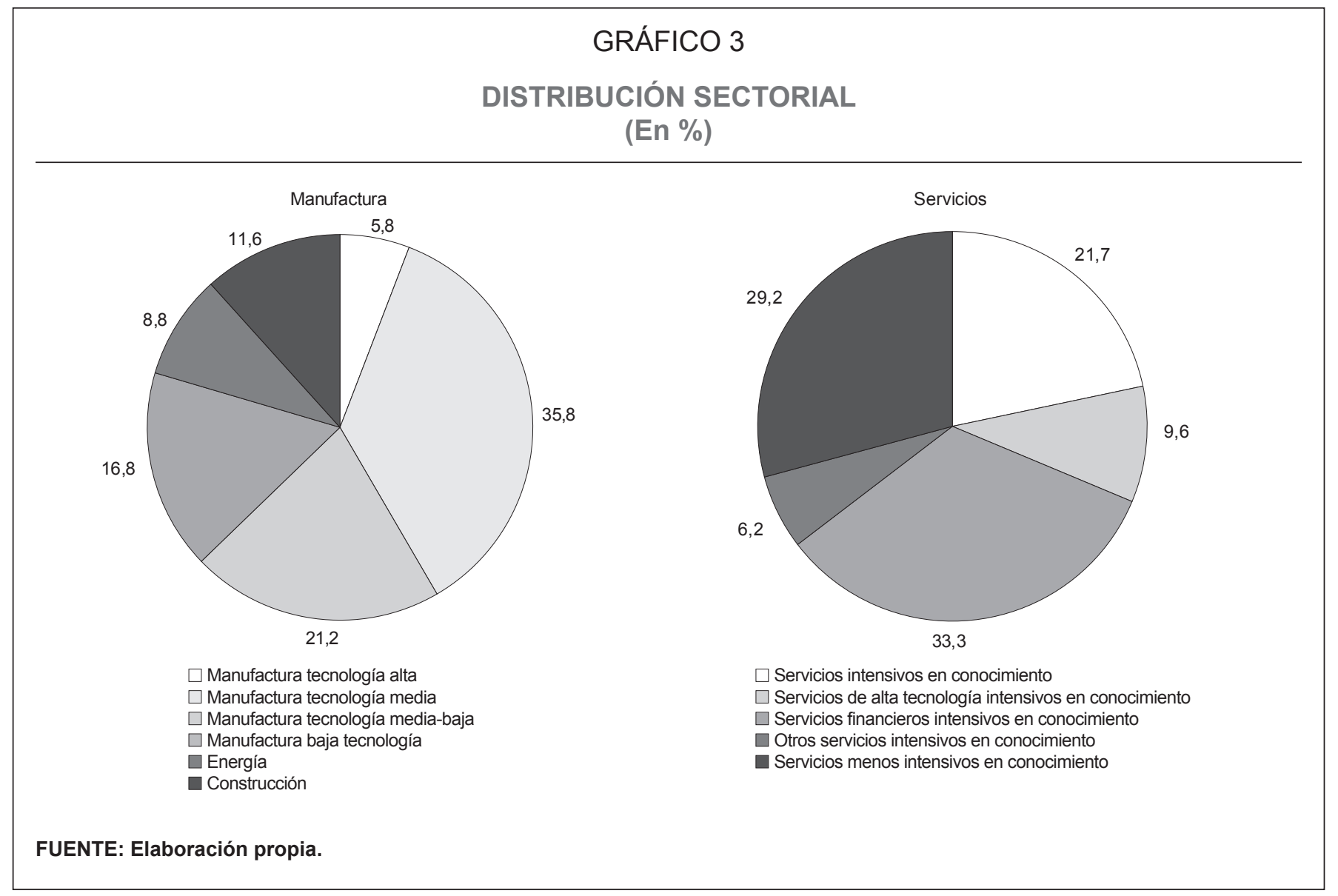

Para ello, utilizamos nuestra muestra de filiales trampolín y las comparamos con el grupo de control.

\section{Medidas de las variables}

A continuación detallamos como han sido medidas las distintas variables. En primer lugar, clasificamos la muestra en dos grupos: las filiales trampolín (69) y las filiales del grupo de control, localizadas en España y cuya casa matriz tenía inversiones en Latinoamérica no gestionadas a través de España (119).

Conocimiento experiencial. La medida del conocimiento experiencial se obtuvo a partir del trabajo seminal de Erikkson et al. (1997). La escala fue adaptada para el caso específico de Latinoamérica e incluyó siete ítems (Cuadro 1).
Disponibilidad de recursos directivos y financieros. La información sobre ambos tipos se obtuvo de fuentes secundarias. La holgura directiva se refiere a la disponibilidad de los gerentes para desplegar esfuerzos en otra actividad. Medimos la disponibilidad de recursos directivos como la relación entre los miembros del equipo de alta dirección en la filial española y el número de empleados en esa unidad en los tres años anteriores al de referencia. Aunque trabajos anteriores (Verbeke y Yuan, 2013) utilizan la productividad de los empleados, creemos que nuestra medida capta mejor el alcance y la calidad del sistema directivo y, en gran medida, la holgura de gestión potencial que podría utilizarse para otras iniciativas. En términos de holgura financiera, utilizamos la relación capital / deuda (Lin et al., 2009; Verbeke y Yuan, 2013). Un nivel más alto de disponibilidad de 


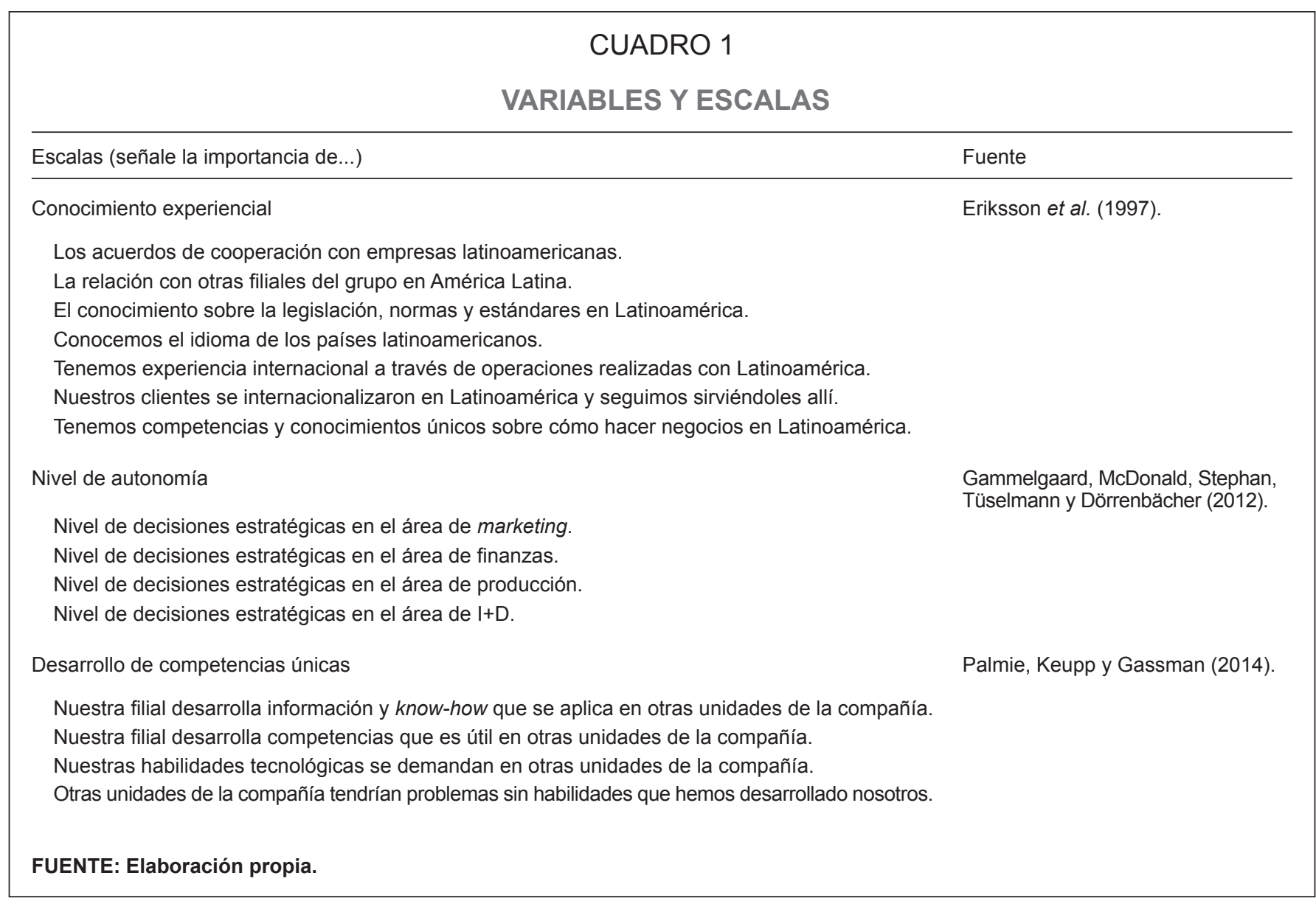

recursos financieros le permite a la filial explorar nuevas oportunidades.

El alcance geográfico de la cadena de valor se refiere a los límites geográficos de la organización, es decir, en qué medida la filial ha extendido sus operaciones y activos internacionales a través de las fronteras. Si bien el alcance geográfico ha sido medido tradicionalmente como el porcentaje de ventas internacionales (por ejemplo, Birkinshaw y Hood, 2000), la literatura más reciente enfatiza el papel de la inversión directa y reconoce las dificultades de hacer negocios fuera de la región de origen (Verbeke y Asmussen, 2016). Por tanto, utilizamos una medida que define el alcance de la filial en términos de una sola región, birregional o global.

El alcance de la actividad de la cadena de valor se refiere a la amplitud de actividades realizadas por la empresa. En el cuestionario solicitamos que se reportasen las actividades que desarrollaban tales como producción, logística, marketing, I+D o ventas. Consideramos que las filiales centradas en un determinado tipo de actividad (ya sea en las etapas iniciales o posteriores del proceso de producción) están especializadas en una fase, mientras que las que realizaban diferentes actividades se codificaron como de amplio alcance.

La capacidad de la filial para generar competencias únicas se mide a través de una escala tipo Likert sobre siete puntos compuesta por cuatro ítems basada en la escala de Palmie, Keupp y Gassman (2014). Los ítems miden la capacidad de la filial para generar conocimiento aplicado en otras unidades de la compañía.

El grado de autonomía se ha utilizado también ampliamente en la investigación sobre las relaciones casa 
CUADRO 2

PRUEBAS U DE MANN-WHITNEY PARA FILIALES TRAMPOLÍN/FILIALES CONTROL

DIFERENCIAS ENTRE FILIALES TRAMPOLÍN Y FILIALES CONTROL

\begin{tabular}{|c|c|c|c|}
\hline & Filiales trampolín & Filiales control & Diferencia de medias (pSig.) \\
\hline Conocimiento experiencial & & & $0,001^{* *}$ \\
\hline $\mathrm{N}$ & 67,00 & 126,00 & \\
\hline Media & 5,10 & 4,70 & \\
\hline Desv. típica & 1,50 & 0,80 & \\
\hline Nivel de autonomía & & & $0,000^{* *}$ \\
\hline $\mathrm{N}$ & 67,00 & 126,00 & \\
\hline Media & 3,20 & 2,20 & \\
\hline Desv. típica & 1,30 & 1,20 & \\
\hline Desarrollo de competencias especiales & & & $0,005^{* *}$ \\
\hline $\mathrm{N}$ & 67,00 & 126,00 & \\
\hline Media & 4,30 & 3,40 & \\
\hline Desv. típica & 2,00 & 1,96 & \\
\hline Disponibilidad de recursos financieros & & & 0,45 \\
\hline $\mathrm{N}$ & 67,00 & 126,00 & \\
\hline Media & 0,35 & 0,30 & \\
\hline Desv. típica & 0,70 & 0,40 & \\
\hline Disponibilidad de recursos directivos & & & $0,013^{*}$ \\
\hline $\mathrm{N}$ & 67.00 & 126,00 & \\
\hline Media & 0,40 & 0,30 & \\
\hline Desv. típica & 0,60 & 0,70 & \\
\hline
\end{tabular}

matriz-filiales. Utilizamos la escala de autonomía estratégica en las áreas de marketing, finanzas, RR.HH., I+D y producción desarrollada por Gammelgaard, McDonald, Stephan, Tüselmann y Dörrenbächer (2012).

En el Cuadro 1 detallamos los ítems que componen las variables medidas con escalas.

\section{Análisis estadístico y resultados}

El objetivo de esta investigación es encontrar las diferencias determinantes de la filial trampolín en comparación con el resto de filiales locales. Para confirmar la diferencia entre dos grupos, utilizamos, por un lado, el test $U$ de Mann-Withney (prueba adecuada para la confirmación de diferencias cuando se trata de variables continuas) y los cuadros de contingencia (adecuada en el caso de relaciones entre variables categóricas).

El Cuadro 2 muestra los resultados del test $\mathrm{U}$ de Mann-Whitney para las hipótesis $H 1, H 2 a, H 2 b$, $H 3 c$ y $H 3 d$ dada la naturaleza continua de las variables asociadas. La $U$ de Mann-Whitney es un test no paramétrico empleado cuando las variables no siguen distribuciones normales y el objetivo es contrastar diferencias entre dos grupos independientes.

Para conocer la dirección de la diferencia entre los grupos reportamos la media para cada variable para el grupo de filiales trampolín y para el resto así como la 


\begin{tabular}{|c|c|c|c|c|c|}
\hline \multicolumn{6}{|c|}{ CUADRO 3} \\
\hline & \multicolumn{5}{|c|}{$\begin{array}{c}\text { CUADRO DE CONTINGENCIA PARA ESPECIALIZACIÓN } \\
\text { Y FILIALES TRAMPOLÍN/FILIALES CONTROL }\end{array}$} \\
\hline & & & Filiales control & Filiales trampolín & Total \\
\hline \multirow[t]{4}{*}{ Especialización de la filial } & Alta (una actividad) & $\mathrm{n}$ & 91,0 & 31,0 & 122 \\
\hline & & $\mathrm{RTC}^{*}$ & 3,6 & $-3,6$ & \\
\hline & Baja (varias actividades) & $\mathrm{n}$ & 35,0 & 36,0 & 71 \\
\hline & & $\mathrm{RTC}^{*}$ & $-3,6$ & 3,6 & \\
\hline Total & & Recuento & 126,0 & 67,0 & 193 \\
\hline
\end{tabular}

\begin{tabular}{|c|c|c|c|c|c|}
\hline \multicolumn{6}{|c|}{$\begin{array}{c}\text { CUADRO DE CONTINGENCIA PARA ALCANCE GEOGRÁFICO } \\
\text { Y FILIALES TRAMPOLÍN/ FILIALES CONTROL }\end{array}$} \\
\hline & & & Filiales control & Filiales trampolín & Total \\
\hline \multirow[t]{6}{*}{ Regiones en las que opera } & Solo región de origen & $\mathrm{n}$ & 77,0 & 0,0 & 77 \\
\hline & & $\mathrm{RTC}^{*}$ & 8,4 & $-8,4$ & \\
\hline & Birregional & $\mathrm{n}$ & 16,0 & 41,0 & 57 \\
\hline & & $\mathrm{RTC}^{*}$ & $-6,9$ & 6,9 & \\
\hline & Global & $\mathrm{n}$ & 28,0 & 25,0 & 53 \\
\hline & & $\mathrm{RTC}^{*}$ & $-2,1$ & 2,1 & \\
\hline \multicolumn{2}{|c|}{$\begin{array}{l}\text { Total } \\
\text { NOTA: *Residuos tipificados corregidos (RTC). } \\
\text { FUENTE: Elaboración propia. }\end{array}$} & Recuento & 120,4 & 66,6 & 187 \\
\hline
\end{tabular}

significatividad del estadístico mediante el $p$-valor. Como podemos observar, existen diferencias significativas entre el grupo de filiales trampolín y el grupo de control para las variables conocimiento experiencial, nivel de autonomía, desarrollo de competencias únicas y nivel de holgura directiva. Por ello, aceptamos la $H 1, H 2 a, H 3 c$ y $H 3 d$. Para todas ellas, la media de las filiales trampolín es superior en línea con nuestras propuestas teóricas. No existen diferencias para el nivel de disponibilidad de recursos financieros y por tanto rechazamos la $\mathrm{H} 2 b$. La filiales trampolín son filiales que cuentan con una elevada autonomía que han sabido desarrollar competencias únicas para que sean aprovechadas por otras unidades de la organización, con un conocimiento sustancial de los mercados latinoamericanos y con cierta holgura directiva que puede utilizarse en la expansión interregional.

Los Cuadros 3 y 4 recogen las relaciones de dependencia entre las filiales trampolín y las filiales de control para el alcance de las actividades de la cadena de valor y el alcance geográfico. En ambos casos se reportan los residuos tipificados corregidos. Si estos residuos presentan un valor en términos absolutos superior 
a 1,96 existe un $95 \%$ de probabilidad de que la asociación entre ambas variables no se deba al azar. En concreto, para un nivel de confianza del 0,95 $(\alpha=0,05)$ aquellos residuos mayores a $\pm 1,96$ indican que en dicha celda se acumulan más casos que los que cabría esperar si las variables fuesen independientes $y$, por tanto, señala una relación de dependencia. Como podemos observar, los residuos tipificados corregidos para ambas variables presentan valores positivos y superiores a 1,96 para las filiales trampolín con respecto a una baja especialización (muchas actividades en la cadena de valor) así como un alcance geográfico más allá de su región de origen (en dos o más regiones). Estos resultados nos permiten aceptar las $H 3 a$ y $H 3 b$.

En este análisis estadístico hemos identificado una serie de recursos y competencias clave que influyen en la adopción del rol de la filial trampolín. De estos recursos aquellos relacionados con la especificidad del conocimiento a nivel de la filial, en línea con la teoría de recursos y capacidades (Grant, 1996), son los más relevantes. Nuestros resultados destacan la relevancia del conocimiento junto con un amplio alcance de valor y geográfico como los elementos determinantes para adoptar este rol de mayor valor estratégico. Este amplio abanico de actividades y dominio enriquece la base de conocimientos de la filial imbuyendo en estas una actitud más emprendedora y activa independientemente de su experiencia internacional.

Por otra parte, nuestros resultados indican que las filiales trampolín son filiales con cierto grado de autonomía en sus decisiones estratégicas así como poseedoras de cierto reconocimiento dentro de la compañía por la creación de conocimiento único.

Todas las características mencionadas reproducen el perfil de una filial con un mandato estratégicamente relevante como por ejemplo, un centro de excelencia (Holm et al., 2000) o las unidades intermedias como una casa matriz regional (Lasserre, 1996). Sin embargo, el papel del conocimiento experiencial en una región distinta de la de origen resulta novedoso en el estudio de las filiales con mandatos relevantes y un diferenciador fundamental en el caso de la filial trampolín.

\section{Conclusiones}

En el presente trabajo hemos analizado una realidad económica que cada vez tiene una mayor relevancia en nuestro país por el creciente y significativo aumento de las relaciones comerciales y diplomáticas con la región latinoamericana: un valor intangible que España puede reivindicar en la esfera global.

El estudio empírico presentado ofrece una visión general del perfil de la filial trampolín y las características asociadas al rol. De este podemos inferir importantes contribuciones en el ámbito de la práctica empresarial. Las filiales trampolín tienen un elevado alcance de actividad y geográfico con un conocimiento sustancial de la realidad latinoamericana. Además han sabido desarrollar cierta autonomía lo que les permite no solo ser reconocidas por el resto de la organización sino además aportar conocimiento específico a otras unidades de la empresa.

Este estudio ofrece una perspectiva distinta en la configuración de la multinacional como red basada en la necesidad de ubicar las funciones de la matriz donde estas se puedan realizar de la manera más eficiente en el proceso de expansión interregional. Estas filiales permiten mejorar la implantación de las empresas multinacionales en Latinoamérica en la medida en que facilitan la adquisición de parte del conocimiento experiencial necesario para abordar dicha región sin la necesidad inicial de invertir en esta. Una innovación organizativa, olvidada por la literatura sobre internacionalización, que permite, en parte, mitigar los problemas a los que se enfrentan las empresas multinacionales cuando invierten en entornos institucionales muy diferentes a los del país de origen y que podría servir como modelo de benchmarking para otras empresas.

Nuestro trabajo muestra la evidencia de una estrategia que incrementa la legitimidad de la inversión en los mercados locales sin la necesidad de realizar costosas 
y complejas inversiones en adquisiciones o negociaciones con empresas en el país de destino. Este hecho es especialmente relevante en la entrada en mercados emergentes, como es el caso de Latinoamérica, en los cuales las empresas de países más desarrollados se enfrentan a instituciones poco reguladas donde existe un mayor riesgo de pérdida de valor. Esta estructura intermedia, con una mayor experiencia y conocimiento en la región, liderará inicialmente el proceso, limitará en cierta medida el riesgo y podrá generar más fácilmente derrames positivos en los mercados locales.

En el análisis descriptivo hemos mostrado como las potenciales ventajas de esta estrategia son especialmente manifiestas en sectores de servicios de empresas europeas, en los que la experiencia española permite acelerar el proceso de aprendizaje en la inversión local, supliendo los problemas derivados de la falta de conocimiento cultural, institucional y empresarial. La posición intermedia de España en términos culturales e institucionales entre Europa y Latinoamérica es clara, y puede ser aprovechada por las empresas europeas, sin embargo entre Asia o Norteamérica y Latinoamérica dicha relación institucional con España ya no es tan evidente. No obstante, si las empresas de estas nacionalidades utilizan esta estrategia podría ser fundamentalmente, no por la búsqueda de las ventajas derivadas de la afinidad cultural e institucional sino por el potencial aprovechamiento de las redes empresariales establecidas en Latinoamérica en determinados sectores en los que España ha mostrado su liderazgo internacional.

A nivel práctico, la filial trampolín es un enfoque indirecto utilizado por las casas matrices para localizar recursos y permanecer envueltos en el desarrollo de competencias y asignación de procesos sin destruir valor. No obstante, consideramos que nuestro concepto de «filial trampolín» funciona mejor para empresas de tamaño medio que no tienen amplias redes de filiales consolidadas por todo el mundo y donde los aspectos de riesgo, legitimidad en el país de destino y tiempo todavía son importantes.
En al análisis cuantitativo hemos evidenciado la importancia de contar con ciertos recursos y competencias específicas para desarrollar este rol. En este sentido, es fundamental disponer de un stock de conocimiento experiencial sobre la región de destino, en este caso, sobre Latinoamérica. Adicionalmente hemos mostrado como la base de nuevos conocimientos, que se generan en aquellas empresas con un amplio alcance geográfico y de actividad, se convierten en competencias específicas que permiten reconocer oportunidades más allá de las fronteras nacionales y por tanto alcanzar roles de mayor importancia estratégica dentro de la multinacional. Una recomendación importante es que estas filiales deben ser capaces de reconocer estas ventajas específicas y potenciarlas, invirtiendo en mayores dotaciones de conocimiento experiencial relevante (tácito y específico), y lo que es más importante, reivindicarlo en la casa matriz y en el resto de filiales de la multinacional. Ello le permitirá solicitar la disposición de recursos adicionales y un mayor grado de autonomía para ejercer las funciones de coordinación, control e, incluso, inversión en otras filiales.

La consolidación de este modelo de estructura intermedia podría tener efectos positivos adicionales en cuanto a la atracción de inversión directa extranjera en la medida en que los ejecutivos de estas filiales fuesen capaces de atraer la atención de sus casas matrices y reivindicar las posibilidades de este nuevo rol de mayor valor. A nivel de sector, estas filiales con un rol más activo no solo garantizan la inversión extranjera en un país sino que generan efectos de derrame positivos hacia los sectores auxiliares.

Aunque nuestro modelo de filial trampolín se ha aplicado a las relaciones específicas entre Europa, España y Latinoamérica, creemos que podría aplicarse a cualquier inversión entre diferentes regiones donde una hipotética estructura intermedia podría actuar como puente entre diferentes unidades de la organización. Así, por ejemplo, desde la práctica empresarial se señalan países como Turquía para la entrada en Oriente Medio, Austria o Polonia para la entrada en 
países de Europa del Este o Finlandia para la entrada en las repúblicas bálticas y Rusia. En este sentido, con las oportunas adaptaciones a cada caso, por ejemplo, la filial podría situarse en la misma región que la red local o incluso en diferentes regiones, el modelo podría ser generalizable y enriquecer una reciente corriente que afirma que invirtiendo en pocos países, que pueden actuar como hubs podría obtenerse una mayor eficiencia y alcance global (Prahalad y Bhattacharyya, 2008).

En definitiva, nuestro estudio analiza un problema económico y social relevante como es la necesaria integración de instituciones, empresas multinacionales y empresas locales para configurar un marco de relaciones que permita fomentar beneficios recíprocos, mejorar las prácticas empresariales y generar una mayor legitimidad de la inversión extranjera.

\section{Referencias bibliográficas}

Alfoldi, E. A., Clegg, L. J., \& McGaughey, S. L. (2012). Coordination at the edge of the empire: The delegation of headquarters functions through regional management mandates. Journal of International Management, 18(3), 276-292.

Andersson, U., \& Forsgren, M. (2000). In search of centre of excellence: Network embeddedness and subsidiary roles in multinational corporations. MIR: Management International Review, 329-350.

Andersson, U., Forsgren, M., \& Holm, U. (2007). Balancing subsidiary influence in the federative MNC: A business network view. Journal of International Business Studies, 38(5), 802-818.

Asmussen, C. G., \& Goerzen, A. (2013). Unpacking dimensions of foreignness: Firm-specific capabilities and international dispersion in regional, cultural, and institutional space. Global Strategy Journal, 3(2), 127-149.

Asmussen, C. G., Foss, N. J., \& Pedersen, T. (2013). Knowledge transfer and accommodation effects in multinational corporations: evidence from European subsidiaries. Journal of Management, 39(6), 1397-1429.

Birkinshaw, J. (1997). Entrepreneurship in multinational corporations: The characteristics of subsidiary initiatives. Strategic Management Journal, 18(3), 207-229.

Birkinshaw, J., Hood, N., \& Jonsson, S. (1998). Building firm-specific advantages in multinational corporations: the role of subsidiary initiative. Strategic Management Journal, 19(3), 221-242.

Birkinshaw, J., \& Hood, N. (2000). Characteristics of foreign subsidiaries in industry clusters. Journal of International Business Studies, 31(1), 141-154.

Birkinshaw, J., Bouquet, C., \& Ambos, T. C. (2007). Managing executive attention in the global company. MIT Sloan Management Review, 48(4), 39.

Campbell, A., \& Szulanski, G. (2016). Knowing when corporate headquarters adds rather than subtracts value. McKinsey Quarterly, January, 1-8.

Cantwell, J., \& Mudambi, R. (2005). MNE competencecreating subsidiary mandates. Strategic Management Journal, 26(12), 1109-1128.

Chakravarty, D., Hsieh, Y., Schotter, A. P. J., \& Beamish, P. (2017). Multinational enterprise regional management centres: Characteristics and performance. Journal of World Business, 52, 296-311.

Economic Commission for Latin America and the Caribbean (ECLAC) (2018). Foreign Direct Investment in Latin America and the Caribbean, (LC/PUB.2018/13-P), Santiago.

Eriksson, K., Johanson, J., Majkgård, A., \& Sharma, D. D. (1997). Experiential knowledge and costs in the internationalization process. Journal of International Business Studies, 28(2), 337-360.

Forsgren, M., Holm, U., \& Johanson, J. (2005). Managing the embedded multinational: A business network view. Edward Elgar Publishing.

Frost, T. S., Birkinshaw, J. M., \& Ensign, P. C. (2002). Centers of excellence in multinational corporations. Strategic Management Journal, 23(11), 997-1018.

Gammelgaard, J., McDonald, F., Stephan, A., Tüselmann, H., \& Dörrenbächer, C. (2012). The impact of increases in subsidiary autonomy and network relationships on performance. International Business Review, 21(6), 1158-1172.

Global LATAM (2018). Series on Foreign Direct Investment. ICEX-Invest in Spain.

Grant, R. M. (1996). Toward a knowledge-based theory of the firm. Strategic Management Journal, 17(2), 109-122.

Gupta, A. K., \& Govindarajan, V. (1991). Knowledge flows and the structure of control within multinational corporations. Academy of Management Review, 16(4), 768-792.

Gupta, A. K., \& Govindarajan, V. (2000). Knowledge flows within multinational corporations. Strategic Management Journal, 21(4), 473-496.

Harzing, A. W., \& Noorderhaven, N. (2006). Knowledge flows in MNCs: An empirical test and extension of Gupta and Govindarajan's typology of subsidiary roles. International Business Review, 15(3), 195-214.

Hoenen, A. K., Nell, P. C., \& Ambos, B. (2014). MNE entrepreneurial capabilities at intermediate levels: the roles of 
external embeddedness and heterogeneous environments. Long Range Planning, 47, 76-86.

Holm, U., Pedersen, T., \& Björkman, I. (2000). The emergence and impact of MNC centres of excellence. Basingstoke, UK.

ICEX (2019). Spain: your partner in Europe. Business Opportunities. ICEX-Invest in Spain.

Johanson, J. \& Vahlne, J. E. (1977). The internationalization process of the firm: a model of knowledge development and increasing foreign market commitments. Journal of International Business Studies, 8, 23-32.

Johanson, J., \& Vahlne, J. E. (2009). The Uppsala internationalization process model revisited: From liability of foreignness to liability of outsidership. Journal of International Business Studies, 40(9), 1411-1431.

Lasserre, P. (1996). Regional headquarters: The spearhead for Asia Pacific markets. Long Range Planning, 29(1), 30-37.

Lin, W. T., Cheng, K. Y., \& Liu, Y. (2009). Organizational slack and firm's internationalization: A longitudinal study of hightechnology firms. Journal of World Business, 44(4), 397-406.

March, J. G. (1991). Exploration and exploitation in organizational learning. Organization Science, 2(1), 71-87.

Mishina, Y., Pollock, T. G., \& Porac, J. (2004). Are more resources always better for growth? Resource stickiness in market and product expansion. Strategic Management Journal, 25, 1179-1197.

Palmié, M., Keupp, M., \& Gassmann, O. (2014). Pull the right levers: Creating internationally "Useful" subsidiary competence by organizational architecture. Long Range Planning, 47, 32-48.

Pla-Barber, J., \& Camps, J. (2012). Springboarding: a new geographical landscape for European foreign investment in Latin America. Journal of Economic Geography, 12, 519-538.
Pla-Barber, J., Villar, C., \& Madhok, A. (2018). Co-parenting through subsidiaries: A model of value creation in the multinational firm. Global Strategy Journal, 8(4), 536-562.

Prahalad, C. K., \& Bhattacharyya, H. (2008). Twenty Hubs and No HQ-A new form of global organization grounded in "gateway" countries can allow a company to operate profitably around the world. Strategy and Business, (50), 24.

Qian, G., Li, L., \& Rugman, A. M. (2013). Liability of country foreignness and liability of regional foreignness: Their effects on geographic diversification and firm performance. Journal of International Business Studies, 44(6), 635-647.

Rugman, A., \& Verbeke, A. (2001). Subsidiary-specific advantages in Multinational Enterprises. Strategic Management Journal, 22(3), 237-250.

Rugman, A. M., \& Verbeke, A. (2003). Extending the theory of the multinational enterprise: Internalization and strategic management perspectives. Journal of International Business Studies, 34(2), 125-137.

Schleimer, S. C., \& Pedersen, T. (2014). The effects of MNC parent effort and social structure on subsidiary absorptive capacity. Journal of International Business Studies, 45(3), 303-320.

Verbeke, A., \& Yuan, W. (2013). The drivers of multinational enterprise subsidiary entrepreneurship in China: A new resource-based view perspective. Journal of Management Studies, 50(2), 236-258.

Verbeke, A., \& Asmussen, C. G. (2016). Global, Local, or Regional? The Locus of MNE Strategies. Journal of Management Studies, 53(6), 1051-1075.

Verbeke, A., \& Yuan, W. (2018). The dynamics of multinational enterprise subsidiary roles in an era of regionalization. In The Routledge Companion to the Geography of International Business (pp. 112-130). Routledge. 


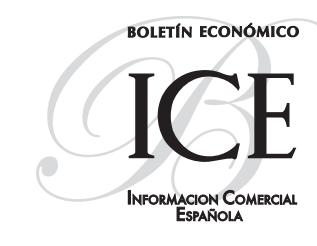

\section{ÚLTIMOS MONOGRÁFICOS PUBLICADOS}
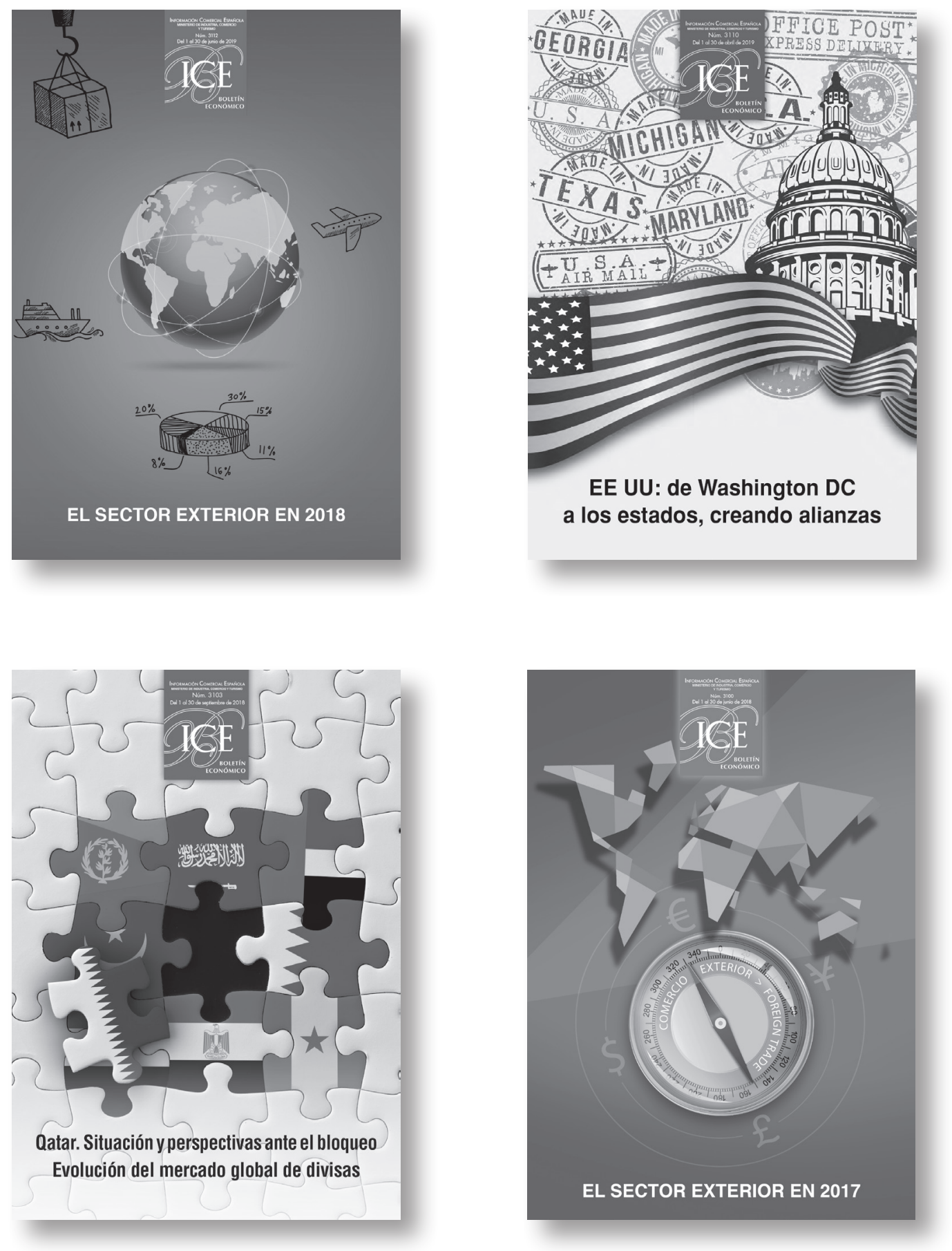

Librería virtual y venta www.mincotur.es

Panamá, 1. 28046 Madrid

Telf. 913495129

www.revistasice.com

CentroPublicaciones@mincotur.es 